EUROPEAN JOURNAL OF PURE AND APPLIED MATHEMATICS

Vol. 13, No. 5, 2020, 1231-1240

ISSN 1307-5543 - www.ejpam.com

Published by New York Business Global

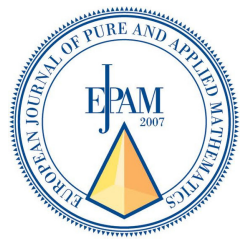

\title{
Special Issue Dedicated to Professor Hari M. Srivastava On the Occasion of his 80th Birthday
}

\section{On the Wiener index of the dot product graph over monogenic semigroups}

\author{
Büşra Aydın ${ }^{1}$, Nihat Akgüneş ${ }^{1, *}$, Ismail Naci Cangul ${ }^{2}$ \\ 1 Department of Mathematics-Computer Sciences, Necmettin Erbakan University, Konya, \\ Turkey \\ ${ }^{2}$ Department of Mathematics, Bursa Uludag University, Bursa, Turkey
}

\begin{abstract}
Algebraic study of graphs is a relatively recent subject which arose in two main streams: One is named as the spectral graph theory and the second one deals with graphs over several algebraic structures. Topological graph indices are widely-used tools in especially molecular graph theory and mathematical chemistry due to their time and money saving applications. The Wiener index is one of these indices which is equal to the sum of distances between all pairs of vertices in a connected graph. The graph over the finite dot product of monogenic semigroups has recently been defined and in this paper, some results on the Wiener index of the dot product graph over monogenic semigroups are given.
\end{abstract}

2020 Mathematics Subject Classifications: 05C12, 05C25

Key Words and Phrases: Dot product, dot product graph, Wiener index, topological index, monogenic semigroup

\section{Introduction and preliminaries}

The connections between graph theory and ring theory was first established in 1988 by Beck [1]. Since then, different types of algebraic graphs have been introduced and studied. The most well-known example is the zero divisor graphs. Anderson and Livingston studied the zero divisor graph of a commutative ring in [2]. Similarly, zero divisor graphs

${ }^{*}$ Corresponding author.

DOI: https://doi.org/10.29020/nybg.ejpam.v13i5.3745

Email addresses: bsrcgn@gmail.com (B. Aydın), nakgunes@erbakan.edu.tr (N. Akgünes), cangul@uludag.edu.tr (I. N. Cangul) 
of commutative semigroups have been studied in [3], [5] and [4]. The zero divisor graphs have been studied intensively, see e.g. [6], [7], [9] and [8]. Further, Badawi has also studied the dot product graphs of commutative rings in [10]. Akgünes has defined the graph of monogenic semigroups in [11] in a similar manner. The dot product graphs of monogenic semigroups have been studied in [12].

Topological graph indices are very useful tools in graph theory and mathematical chemistry. By modelling a chemical substance with a graph, we can apply these indices and obtain physico-chemical properties of that substance solely by means of mathematical calculations without any experiments in laboratory. The Wiener index is one of the significant and surely the oldest topological indices used in mathematical chemistry. It was introduced in 1947 by Wiener [13] and used for modelling the shape of organic molecules and for calculating several of their physico-chemical properties, in particular the boiling points of alkane isomers. Mathematicians are interested in the Wiener index as much as chemists. Mathematical research on the Wiener index was started in 1976 in [14] and since then, this distance-based quantity had been studied in [16], [17] and [15].

Now let us recall the definitions of the Wiener index and monogenic semigroups:

The vertex set of a graph $G$ is denoted by $V(G)$. The distance between two vertices $u$ and $v$ in $V(G)$ is denoted by $d_{G}(u, v)$. Then the Wiener index $W(G)$ of $G$ is defined by

$$
W(G)=\sum_{\{u, v\} \subseteq V(G)} d_{G}(u, v) .
$$

Let $S^{n}$ be a finite semigroup of order $n$. In [11], an undirected graph denoted by $\left\lceil\left(S^{n}\right)=(V, E)\right.$ was defined as follows: The vertex set consists of the non-zero elements of $S^{n}$ and two vertices $x$ and $y$ which are distinct non-zero elements in $S^{n}$ are adjacent if and only if $x y=0_{S^{n}}$. Hence

$$
S^{n}=\left\{0, x, x^{2}, \cdots, x^{n}\right\}
$$

where $x^{i}, x^{j} \in V\left(\left\lceil\left(S^{n}\right)\right)\right.$ are adjacent $\Leftrightarrow x^{i} \cdot x^{j}=0_{S^{n}} \Leftrightarrow x^{i+j}=0_{S^{n}} \Leftrightarrow i+j>n$ $(1 \leq i, j \leq n)$.

Some various types of monogenic semigroups including the tensor, lexicographic, strong and disjunctive products were studied in [20], [21], [19] and [18]. Also some topological indices of monogenic semigroups were calculated in [24], [22] and [23].

In [12], the dot product graph considered was $\lceil(S)$ where $S$ is the cartesian product of $k$ times $S^{n}$ :

$$
S=S^{n} \times S^{n} \times \ldots \times S^{n} \text { for } k \text { times, where } 1 \leq k<\infty .
$$


Let two nonzero elements of $S$ be $X=\left(x^{i_{1}}, x^{i_{2}}, \cdots, x^{i_{k}}\right)$ and $Y=\left(x^{j_{1}}, x^{j_{2}}, \cdots, x^{j_{k}}\right)$ for $\left\{i_{t}\right\}_{t=1}^{k},\left\{j_{t}\right\}_{t=1}^{k} \in\{0,1,2, \cdots, n\}$ where $x^{i_{t}}=0_{S^{n}}$ if and only if $i_{t}=0$. A more explicit definition of the dot product in terms of the elements of $S$ is as follows:

$$
\begin{aligned}
X \cdot Y & =\left(x^{i_{1}}, x^{i_{2}}, \cdots, x^{i_{k}}\right) \cdot\left(x^{j_{1}}, x^{j_{2}}, \cdots, x^{j_{k}}\right) \\
& =x^{i_{1}} \cdot x^{j_{1}}+x^{i_{2}} \cdot x^{j_{2}}+\cdots+x^{i_{k}} \cdot x^{j_{k}}=x^{i_{1}+j_{1}}+x^{i_{2}+j_{2}}+\cdots+x^{i_{k}+j_{k}} .
\end{aligned}
$$

Then the dot product graph $\lceil(S)$ can be defined as an (undirected) graph with vertices $X, Y \in S^{*}=S \backslash\left\{0_{S}\right\}$ such that $X$ and $Y$ are adjacent iff $X \cdot Y=0_{S^{n}}$ and this is denoted by $X \sim Y$.

The following result will be used in the rest of the paper:

Lemma 1 ([12]). $\lceil(S)$ is always connected and its diameter is equal to 2, that is

$$
\operatorname{diam}(\lceil(S))=2 .
$$

\section{Main results}

Let $n \in \mathbb{N}$ and let $S^{n}=\left\{0, x, x^{2}, \cdots, x^{n}\right\}$ be a non-empty monogenic semigroup. Let $\left\lceil(S)\right.$ be the dot product graph where $S=S^{n} \times S^{n}$ is the cartesian product of two $S^{n}$ 's. Recall that its vertices are the non-zero elements in $S$. The following result is clear as the diameter of $\lceil(S)$ is equal to 2 by Lemma 1 :

Lemma $2([12])$. The distance of any two vertices is less than or equal to 2 . That is,

$$
d_{\lceil(S)}\left(v_{i}, v_{j}\right) \leq 2
$$

where $i, j \in \mathbb{N}, v_{i}, v_{j} \in V(\lceil(S))$.

Theorem 1. The Wiener index of the dot product graph over a monogenic semigroup of order $n$ is that

$W(S)=\frac{1}{2}\left[\begin{array}{c}(6 n-2)\left(n^{2}+2 n\right)+\left(\left\lceil\frac{n}{2}\right\rceil+1\right)^{2}-1-\sum_{\substack{k, t \in\{0,1, \ldots, n-1\} \\ k \in\{0,1, \ldots, n-1\} \\ t=n}}[(n-k)(n+1)+n]-\sum_{\substack{t \in\{0,1, \ldots, n-1\} \\ k=n}}[(n-t)(n+1)+n]\end{array}\right]$.

Proof. Since the diameter of $\left\lceil(S)\right.$ is equal to 2 , we can write $d_{\lceil(S)}\left(v_{i}, v_{j}\right) \leq 2$ for every pair $i, j \in \mathbb{N}$ and $v_{i}, v_{j} \in V(\lceil(S))$. Hence the distance of any two vertices is 1 or 2 . Consequently the Wiener index of $\lceil(S)$ is as follows:

$$
W\left(\lceil(S))=\frac{1}{2} \sum_{\left\{v_{i}, v_{j}\right\} \subseteq V(\lceil(S))} d_{\lceil(S)}\left(v_{i}, v_{j}\right) .\right.
$$


Here, let

$$
V_{1}=\left\{v_{i}: d_{\lceil(S)}\left(v_{i}, v_{j}\right)=1, i, j \in \mathbb{N}, v_{j} \in V(\lceil(S))\}\right.
$$

and

$$
V_{2}=\left\{v_{i}: d_{\lceil(S)}\left(v_{i}, v_{j}\right)=2, i, j \in \mathbb{N}, v_{j} \in V(\lceil(S))\} .\right.
$$

Then the Wiener index formula becomes

$$
W\left(\lceil(S))=\frac{1}{2} \sum_{v \in V(\lceil(S))}\left[1 \cdot\left|V_{1}\right|+2 \cdot\left|V_{2}\right|\right] .\right.
$$

So if we find the cardinalities of the sets $V_{1}$ and $V_{2}$, the Wiener index of $\lceil(S)$ will be calculated. Since the distance of any two vertices in $\left\lceil(S)\right.$ is 1 or 2 , it is clear that $\left|V_{2}\right|=$ $|V|-\left|V_{1}\right|$. Then

$$
\begin{aligned}
W(\lceil(S)) & =\frac{1}{2} \sum_{v \in V(\lceil(S))}\left[1 \cdot\left|V_{1}\right|+2 \cdot\left|V_{2}\right|\right] \\
& =\frac{1}{2} \sum_{v \in V(\lceil(S))}\left[1 \cdot\left|V_{1}\right|+2 \cdot\left(|V|-\left|V_{1}\right|\right)\right] \\
& =\frac{1}{2} \sum_{v \in V(\lceil(S))}\left[1 \cdot\left|V_{1}\right|+2 \cdot|V|-2 \cdot\left|V_{1}\right|\right] \\
& =\frac{1}{2} \sum_{v \in V(\lceil(S))}\left[2 \cdot|V|-\left|V_{1}\right|\right]
\end{aligned}
$$

and therefore finding $\left|V_{1}\right|$ will be enough.

In the next step, let us determine the distance of each vertex to other vertices. If we can determine how many distances are 1 , it means that we also determined the number of elements of the set $V_{1}$.

Any pair of two non-zero elements of $S$ will be denoted by $\left(x^{n-k}, x^{n-t}\right)$ where $k, t \in$ $\{0,1,2, \cdots, n\}$. As a special case, $x^{0}=0$ is assumed. Let us now look at the three cases where $k, t \in\{0,1,2, \cdots, n-1\}$ or $k=n$ or $t=n$, respectively.

First case: Let $k, t \in\{0,1,2, \cdots, n-1\}$. There are $(n-k)(n-t)+2 n-k-t$ vertices where the distance of the vertex $\left(x^{n-k}, x^{n-t}\right)$ to another vertex $\left(x^{a}, x^{b}\right)$ in $V(\lceil(S))$ is 1 .

Indeed, any vertex $X=\left(x^{a}, x^{b}\right)$ in $V\left(\lceil(S))\right.$ is adjacent to $Y=\left(x^{n-k}, x^{n-t}\right)$ for $k, t \in\{0,1,2, \cdots, n-1\}$ if $X \cdot Y=0$, so $x^{n-k+a}+x^{n-t+b}=0$. This equality holds if $n-k+a>n$ or $a=0_{S^{n}}$ and $n-t+b>n$ or $a=0_{S^{n}}$.

Alternatively, all elements of $S$ can be thought to form a matrix $[C]_{(n+1) \times(n+1)}$ for $n \in \mathbb{N}$, in $[12]:$ 
B. Aydın, N. Akgüneş, I. N. Cangul / Eur. J. Pure Appl. Math, 13 (5) (2020), 1231-1240

$$
[C]_{(n+1) \times(n+1)}=\left[\begin{array}{ccccccc} 
& \ldots & \mathbf{c}_{0(t+1)} & \mathbf{c}_{0(t+2)} & \ldots & \mathbf{c}_{0(n-1)} & \mathbf{c}_{0 n} \\
\vdots & \ldots & \vdots & \vdots & \ldots & \vdots & \vdots \\
\mathbf{c}_{(k+1) 0} & \ldots & \mathbf{c}_{(k+1)(t+1)} & \mathbf{c}_{(k+1)(t+2)} & \ldots & \mathbf{c}_{(k+1)(n-1)} & \mathbf{c}_{(k+1) n} \\
\mathbf{c}_{(k+2) 0} & \ldots & \mathbf{c}_{(k+2)(t+1)} & \mathbf{c}_{(k+2)(t+2)} & \ldots & \mathbf{c}_{(k+2)(n-1)} & \mathbf{c}_{(k+2) n} \\
\vdots & \ldots & \vdots & \vdots & \ldots & \vdots & \vdots \\
\mathbf{c}_{(n-1) 0} & \ldots & \mathbf{c}_{(n-1)(t+1)} & \mathbf{c}_{(n-1)(t+2)} & \ldots & \mathbf{c}_{(n-1)(n-1)} & \mathbf{c}_{(n-1) n} \\
\mathbf{c}_{n 0} & \ldots & \mathbf{c}_{n(t+1)} & \mathbf{c}_{n(t+2)} & \ldots & \mathbf{c}_{n(n-1)} & \mathbf{c}_{n n}
\end{array}\right]
$$

For $x^{a}$, the values of $a$ may be placed in the zero-row and also in the rows $(k+1)$ to $n$.

For $x^{b}$, the values of $b$ may be placed in the zero-column and also in the columns $(t+1)$ to $n$.

Let us find out the number of vertices at their intersection: $(n-(k+1)+1)+(n-(t+1)+1)+$ $(n-(k+1)+1)(n-(t+1)+1)=2 n-k-t+(n-k)(n-t)=2 n-k-t+(n-k)(n-t)$ vertex pairs have distance 1 .

Second case: Let $k=n$. Here $d\left(\left(x^{0}, x^{n-t}\right),\left(x^{a}, x^{b}\right)\right)=d\left(\left(0, x^{n-t}\right),\left(x^{a}, x^{b}\right)\right)=1$ so there are $(n-t)(n+1)+n$ vertices.

Indeed, if $k=n$ and the vertices $X=\left(0, x^{n-t}\right)$ and $Y=\left(x^{a}, x^{b}\right)$ are adjacent, then $0+x^{n-t+b}=0$. So $n-t+b>n$ or $b=0_{S^{n}}$. Hence similarly to the previous case, we have

$$
[C]_{(n+1) \times(n+1)}=\left[\begin{array}{ccccccc} 
& \ldots & \mathbf{c}_{0(t+1)} & \mathbf{c}_{0(t+2)} & \ldots & \mathbf{c}_{0(n-1)} & \mathbf{c}_{0 n} \\
\vdots & \ldots & \vdots & \vdots & \ldots & \vdots & \vdots \\
\mathbf{c}_{(k+1) 0} & \ldots & \mathbf{c}_{(k+1)(t+1)} & \mathbf{c}_{(k+1)(t+2)} & \ldots & \mathbf{c}_{(k+1)(n-1)} & \mathbf{c}_{(k+1) n} \\
\mathbf{c}_{(k+2) 0} & \ldots & \mathbf{c}_{(k+2)(t+1)} & \mathbf{c}_{(k+2)(t+2)} & \ldots & \mathbf{c}_{(k+2)(n-1)} & \mathbf{c}_{(k+2) n} \\
\vdots & \ldots & \vdots & \vdots & \ldots & \vdots & \vdots \\
\mathbf{c}_{(n-1) 0} & \ldots & \mathbf{c}_{(n-1)(t+1)} & \mathbf{c}_{(n-1)(t+2)} & \ldots & \mathbf{c}_{(n-1)(n-1)} & \mathbf{c}_{(n-1) n} \\
\mathbf{c}_{n 0} & \ldots & \mathbf{c}_{n(t+1)} & \mathbf{c}_{n(t+2)} & \ldots & \mathbf{c}_{n(n-1)} & \mathbf{c}_{n n}
\end{array}\right]
$$

For $x^{b}$, the values of $b$ may be placed in the zero-column and also in the columns $(t+1)$ to $n$. So $(n-(t+1)+1)(n+1)+n=(n-t)(n+1)+n=(n-t)(n+1)+n$ vertex pairs have distance 1 .

Third case: Let $t=n$. Now $d\left(\left(x^{n-k}, x^{0}\right),\left(x^{a}, x^{b}\right)\right)=d\left(\left(x^{n-k}, 0\right),\left(x^{a}, x^{b}\right)\right)=1$, so there are $(n-k)(n+1)+n$ vertices.

In this case, if $t=n$ and $X=\left(x^{n-k}, 0\right)$ and $Y=\left(x^{a}, x^{b}\right)$ vertices are adjacent then $x^{n-k+b}+0=0$. So $n-k+b>n$ or $a=0_{S^{n}}$. Similarly to the first case, we obtain 


$$
[C]_{(n+1) \times(n+1)}=\left[\begin{array}{ccccccc} 
& \ldots & \mathbf{c}_{0(t+1)} & \mathbf{c}_{0(t+2)} & \ldots & \mathbf{c}_{0(n-1)} & \mathbf{c}_{0 n} \\
\vdots & \ldots & \vdots & \vdots & \ldots & \vdots & \vdots \\
\mathbf{c}_{(k+1) 0} & \ldots & \mathbf{c}_{(k+1)(t+1)} & \mathbf{c}_{(k+1)(t+2)} & \ldots & \mathbf{c}_{(k+1)(n-1)} & \mathbf{c}_{(k+1) n} \\
\mathbf{c}_{(k+2) 0} & \ldots & \mathbf{c}_{(k+2)(t+1)} & \mathbf{c}_{(k+2)(t+2)} & \ldots & \mathbf{c}_{(k+2)(n-1)} & \mathbf{c}_{(k+2) n} \\
\vdots & \ldots & \vdots & \vdots & \ldots & \vdots & \vdots \\
\mathbf{c}_{(n-1) 0} & \ldots & \mathbf{c}_{(n-1)(t+1)} & \mathbf{c}_{(n-1)(t+2)} & \ldots & \mathbf{c}_{(n-1)(n-1)} & \mathbf{c}_{(n-1) n} \\
\mathbf{c}_{n 0} & \ldots & \mathbf{c}_{n(t+1)} & \mathbf{c}_{n(t+2)} & \ldots & \mathbf{c}_{n(n-1)} & \mathbf{c}_{n n}
\end{array}\right]
$$

For $x^{a}$, the values of $a$ are placed in zero-row and also in the rows $(k+1)$ to $n$. So $(n-(k+1)+1)(n+1)+n=(n-k-1+1)(n+1)+n=(n-k)(n+1)+n$ vertex pairs have distance 1 .

Let us be careful about the fact that we have not yet excluded the vertices which are adjacent to themselves and the distances between such pairs of vertices do not contribute to Wiener index. So these values should be taken away from the sum we have just found.

Now, let us find how many vertices are adjacent to itself: There are $\left(n-\left\lfloor\frac{n}{2}\right\rfloor\right)^{2}+$ $2\left(n-\left\lfloor\frac{n}{2}\right\rfloor\right)$ vertices with distance 1 to itself. Hence there are $|V|-\left[\left(n-\left\lfloor\frac{n}{2}\right\rfloor\right)^{2}+2\left(n-\left\lfloor\frac{n}{2}\right\rfloor\right)\right]$ vertices having distance 2 to itself. When $k, t \in\left\{0,1,2, \cdots,\left\lceil\frac{n}{2}\right\rceil-1,\left\lceil\frac{n}{2}\right\rceil,\left\lceil\frac{n}{2}\right\rceil+1, \cdots, n-1\right\}$, if any vertex $X=\left(x^{n-k}, x^{n-t}\right)$ is adjacent to itself, then $X \cdot X=0$ so that $x^{2(n-k)}+$ $x^{2(n-t)}=0$. This equality holds when $2(n-k)>n$ or $k=n$ and $2(n-t) n$ or $t=n$. It means that $2 n-2 k>n$ or $k=n$ and $2 n-2 t>n$ or $t=n$ and equivalently $n>2 k$ or $k=n$ and $n>2 t$ or $t=n$. This is equivalent to $k<\frac{n}{2}$ or $k=n$ and $t<\frac{n}{2}$ or $t=n$. Therefore, we get

$$
d\left(\left(x^{n-k}, x^{n-t}\right),\left(x^{n-k}, x^{n-t}\right)\right)=1 \Leftrightarrow k \in\left\{0,1,2, \cdots,\left\lceil\frac{n}{2}\right\rceil-1, n\right\}
$$

and

$$
t \in\left\{0,1,2, \cdots,\left\lceil\frac{n}{2}\right\rceil-1, n\right\} .
$$

So $(n-k) \in\left\{n, n-1, \cdots,\left\lfloor\frac{n}{2}\right\rfloor+1,0\right\}$ and $(n-t) \in\left\{n, n-1, \cdots,\left\lfloor\frac{n}{2}\right\rfloor+1,0\right\}$ since $n-\left\lceil\frac{n}{2}\right\rceil=\left\lfloor\frac{n}{2}\right\rfloor$. In this case, we obtain the following vertices

$$
[C\rfloor_{(n+1) \times(n+1)}=\left[\begin{array}{ccccccc} 
& \cdots & \mathbf{c}_{0\left(\left\lfloor\frac{n}{2}\right\rfloor+1\right)} & \mathbf{c}_{0\left(\left\lfloor\frac{n}{2}\right\rfloor+2\right)} & \cdots & \mathbf{c}_{0(n-1)} & \mathbf{c}_{0 n} \\
\vdots & \cdots & \vdots & \vdots & \cdots & \vdots & \vdots \\
c_{\left(\left\lfloor\frac{n}{2}\right\rfloor+1\right) 0} & \cdots & \mathbf{c}_{\left(\left\lfloor\frac{n}{2}\right\rfloor+1\right)\left(\left\lfloor\frac{n}{2}\right\rfloor+1\right)} & \mathbf{c}_{\left(\left\lfloor\frac{n}{2}\right\rfloor+1\right)\left(\left\lfloor\frac{n}{2}\right\rfloor+2\right)} & \cdots & \mathbf{c}_{\left(\left\lfloor\frac{n}{2}\right\rfloor+1\right)(n-1)} & \mathbf{c}_{\left(\left\lfloor\frac{n}{2}\right\rfloor+1\right) n} \\
c_{\left(\left\lfloor\frac{n}{2}\right\rfloor+2\right) 0} & \cdots & \mathbf{c}_{\left(\left\lfloor\frac{n}{2}\right\rfloor+2\right)\left(\left\lfloor\frac{n}{2}\right\rfloor+1\right)} & \mathbf{c}_{\left(\left\lfloor\frac{n}{2}\right\rfloor+2\right)\left(\left\lfloor\frac{n}{2}\right\rfloor+2\right)} & \cdots & \mathbf{c}_{\left(\left\lfloor\frac{n}{2}\right\rfloor+2\right)(n-1)} & \mathbf{c}_{\left(\left\lfloor\frac{n}{2}\right\rfloor+2\right) n} \\
\vdots & \cdots & \vdots & \vdots & \cdots & \vdots & \vdots \\
c_{(n-1) 0} & \cdots & \mathbf{c}_{(n-1)\left(\left\lfloor\frac{n}{2}\right\rfloor+1\right)} & \mathbf{c}_{(n-1)\left(\left\lfloor\frac{n}{2}\right\rfloor+2\right)} & \cdots & \mathbf{c}_{(n-1)(n-1)} & \mathbf{c}_{(n-1) n} \\
c_{n 0} & \cdots & \mathbf{c}_{n\left(\left\lfloor\frac{n}{2}\right\rfloor+1\right)} & \mathbf{c}_{n\left(\left\lfloor\frac{n}{2}\right\rfloor+2\right)} & \cdots & \mathbf{c}_{n(n-1)} & \mathbf{c}_{n n}
\end{array}\right] .
$$


Thus there are $\left(n-\left\lfloor\frac{n}{2}\right\rfloor\right)^{2}+2\left(n-\left\lfloor\frac{n}{2}\right\rfloor\right)$ vertices with a distance of 1 to itself. Hence there are $|V|-\left[\left(n-\left\lfloor\frac{n}{2}\right\rfloor\right)^{2}+2\left(n-\left\lfloor\frac{n}{2}\right\rfloor\right)\right]$ vertices with a distance of 2 to itself.

Considering the above claims, we obtain the following result:

Since $V_{1}=\left\{v_{i}: v_{i}=\left(x^{a}, x^{b}\right),\left(x^{a}, x^{b}\right) \sim\left(x^{n-k}, x^{n-t}\right), k, t \in\{0,1,2, \ldots, n\}\right\}$, we find

$$
\begin{aligned}
\left|V_{1}\right| & =\sum_{\substack{k, t \in\{0,1, \ldots, n-1\} \\
k \in\{0,1, \ldots, n-1\}}}[2 n-k-t+(n-k)(n-t)] \\
& +\sum_{\substack{t \in\{0,1, \ldots, n-1\} \\
k=n}}[(n-t)(n+1)+n] .
\end{aligned}
$$

Consequently, we conclude that

$$
\begin{aligned}
& W(\Gamma(S))=\frac{1}{2} \sum_{i, j \in \mathbb{N}} d_{\Gamma(S)}\left(v_{i}, v_{j}\right) \\
& =\frac{1}{2}\left[\begin{array}{l}
\sum_{v_{i} \in V([(S))}\left[2 \cdot|V|-\left|V_{1}\right|\right]-1 \cdot\left[\left(n-\left\lfloor\frac{n}{2}\right\rfloor\right)^{2}+2\left(n-\left\lfloor\frac{n}{2}\right\rfloor\right)\right] \\
-2 \cdot\left[|V|-\left(\left(n-\left\lfloor\frac{n}{2}\right\rfloor\right)^{2}+2\left(n-\left\lfloor\frac{n}{2}\right\rfloor\right)\right)\right]
\end{array}\right] \\
& =\frac{1}{2} \sum_{k, t \in\{0,1, \ldots, n-1\}}[2|V|-(n-k)-(n-t)-(n-k)(n-t)]
\end{aligned}
$$

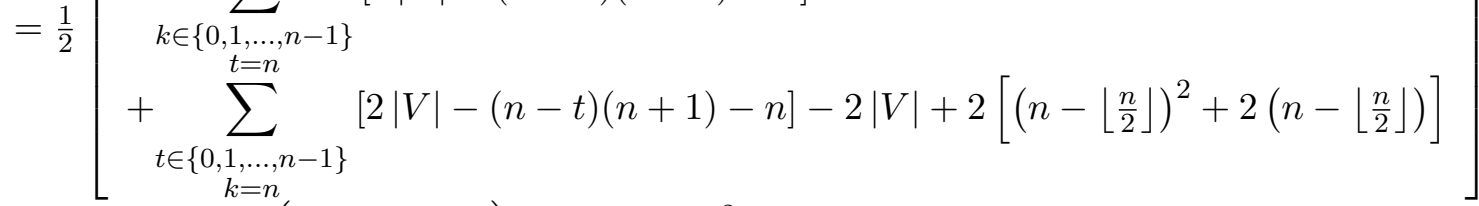

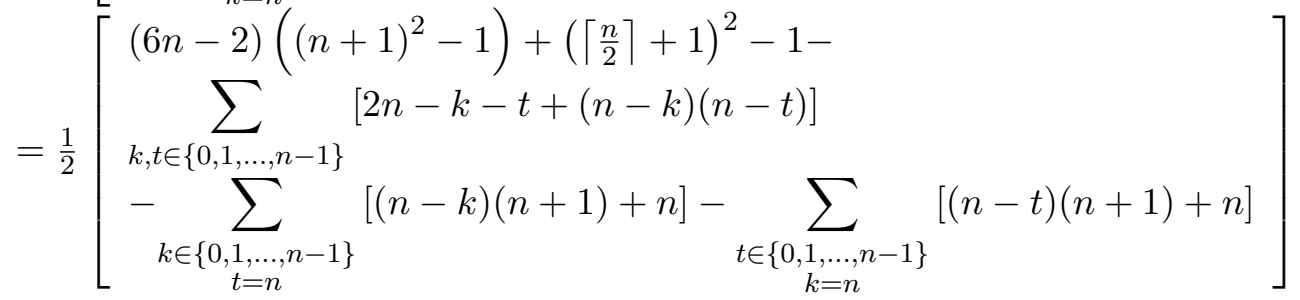

since $|V|=(n+1)^{2}-1$ and $n-\left\lfloor\frac{n}{2}\right\rfloor=\left\lceil\frac{n}{2}\right\rceil$.

\section{Examples}

Let us give the following examples to strengthen the theory. 
Example 1. Let $n=5$. Then $S=S^{5} \times S^{5}$ where $S^{5}=\left\{0, x, x^{2}, \ldots, x^{5}\right\}$. Then we can list the vertices of $S$ as follows:

$$
V(S)=\left\{\begin{array}{cccccc} 
& (0, x) & \left(0, x^{2}\right) & \left(0, x^{3}\right) & \left(0, x^{4}\right) & \left(0, x^{5}\right) \\
(x, 0) & (x, x) & \left(x, x^{2}\right) & \left(x, x^{3}\right) & \left(x, x^{4}\right) & \left(x, x^{5}\right) \\
\left(x^{2}, 0\right) & \left(x^{2}, x\right) & \left(x^{2}, x^{2}\right) & \left(x^{2}, x^{3}\right) & \left(x^{2}, x^{4}\right) & \left(x^{2}, x^{5}\right) \\
\left(x^{3}, 0\right) & \left(x^{3}, x\right) & \left(x^{3}, x^{2}\right) & \left(x^{3}, x^{3}\right) & \left(x^{3}, x^{4}\right) & \left(x^{3}, x^{5}\right) \\
\left(x^{4}, 0\right) & \left(x^{4}, x\right) & \left(x^{4}, x^{2}\right) & \left(x^{4}, x^{3}\right) & \left(x^{4}, x^{4}\right) & \left(x^{4}, x^{5}\right) \\
\left(x^{5}, 0\right) & \left(x^{5}, x\right) & \left(x^{5}, x^{2}\right) & \left(x^{5}, x^{3}\right) & \left(x^{5}, x^{4}\right) & \left(x^{5}, x^{5}\right)
\end{array}\right\} .
$$

Let now $k=4$ and $t=4$. The values $\left(x^{a}, x^{b}\right)$ satisfying the condition

$$
d_{\Gamma(S)}\left(\left(x^{5-4}, x^{5-4}\right),\left(x^{a}, x^{b}\right)\right)=d_{\Gamma(S)}\left((x, x),\left(x^{a}, x^{b}\right)\right)=1
$$

are

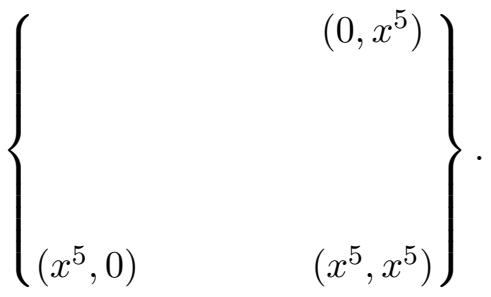

Hence there are

$$
(n-k)(n-t)+2 n-k-t=(5-4)(5-4)+2 \cdot 5-4-4=1 \cdot 1+1+1=3
$$

vertices.

Let us see the same example for another values of $k$ and $t$ : Let $k=1$ and $t=2$. Then the pairs of vertices $\left(x^{a}, x^{b}\right)$ which satisfy the condition

$$
d_{\Gamma(S)}\left(\left(x^{5-1}, x^{5-2}\right),\left(x^{a}, x^{b}\right)\right)=d_{\Gamma(S)}\left(\left(x^{4}, x^{3}\right),\left(x^{a}, x^{b}\right)\right)=1
$$

are listed as

$$
\left\{\begin{array}{llll} 
& \left(0, x^{3}\right) & \left(0, x^{4}\right) & \left(0, x^{5}\right) \\
\left(x^{2}, 0\right) & \left(x^{2}, x^{3}\right) & \left(x^{2}, x^{4}\right) & \left(x^{2}, x^{5}\right) \\
\left(x^{3}, 0\right) & \left(x^{3}, x^{3}\right) & \left(x^{3}, x^{4}\right) & \left(x^{3}, x^{5}\right) \\
\left(x^{4}, 0\right) & \left(x^{4}, x^{3}\right) & \left(x^{4}, x^{4}\right) & \left(x^{4}, x^{5}\right) \\
\left(x^{5}, 0\right) & \left(x^{5}, x^{3}\right) & \left(x^{5}, x^{4}\right) & \left(x^{5}, x^{5}\right)
\end{array}\right\} .
$$

Since, in the above example, $n=5, k=1$ and $t=2$, we obtain the number of vertices satisfying this condition as

$$
(n-k)(n-t)+2 n-k-t=(5-1)(5-2)+2 \cdot 5-1-2=4 \cdot 3+4+3=19 .
$$


So for $n=5$, the vertices adjacent to itself, in other words, the vertices whose distance to itself is 1 can be listed as follows:

$$
\left\{\begin{array}{llll} 
& \left(0, x^{3}\right) & \left(0, x^{4}\right) & \left(0, x^{5}\right) \\
\left(x^{3}, 0\right) & & & \\
\left(x^{4}, 0\right) & \left(x^{3}, x^{3}\right) & \left(x^{3}, x^{4}\right) & \left(x^{3}, x^{5}\right) \\
\left(x^{5}, 0\right) & \left(x^{4}, x^{3}\right) & \left(x^{4}, x^{4}\right) & \left(x^{4}, x^{5}\right) \\
\left(x^{5}, x^{3}\right) & \left(x^{5}, x^{4}\right) & \left(x^{5}, x^{5}\right)
\end{array}\right\} .
$$

The total number of these vertex pairs is 15 which can also be found by the formula $\left(n-\left\lfloor\frac{n}{2}\right\rfloor\right)^{2}+2\left(n-\left\lfloor\frac{n}{2}\right\rfloor\right)=\left(5-\left\lfloor\frac{5}{2}\right\rfloor\right)^{2}+2\left(5-\left\lfloor\frac{5}{2}\right\rfloor\right)=(5-2)^{2}+2(5-2)=3^{2}+2 \cdot 3=9+6=15$ vertices.

Consequently, the Wiener index of the $\Gamma\left(S^{5} \times S^{5}\right)$ which is equal to 895, can also be found by the above formula.

\section{References}

[1] I Beck. Coloring of Commutating Ring. J. Algebra, 116:208-226, 1988.

[2] D F Anderson, P S Livingston. The Zero-Divisor Graph of a Commutative Ring. Journal of Algebra, 217:434-447, 1999.

[3] F R De Meyer, L De Meyer. Zero-Divisor Graphs of Semigroups. J. Algebra, 283:190$198,2005$.

[4] L De Meyer, L Greve, A Sabbaghi, J Wang. The Zero-Divisor Graph Associated to a Semigroup. Communications in Algebra, 38(9):3370-3391, 2010.

[5] F R De Meyer, T Mc Kenzie, K Schneider. The Zero-Divisor Graph of a Commutative Semigroup. Semigroup Forum, 65:206-214, 2002.

[6] S Akbari, H R Maimani, S Yassemi. When a Zero-Divisor Graph is Planar or a Complete r-Partite Graph. J. Algebra, 270:169-180, 2003.

[7] S Akbari, A Mohammadian. On the Zero-Divisor Graph of a Commutative Ring. Journal of Algebra, 274:847-855, 2004.

[8] S E Wright. Lengths of paths and cycles in zero-divisor graphs and digraphs of semigroups. Comm. Algebra, 35:1987-1991, 2007.

[9] R Belsho, J Chapman. Planar Zero-Divisor Graphs. J. Algebra, 316:471-480, 2007. 
[10] A Badawi. On the Dot Product Graph of a Commutative Ring. Communications in Algebra, 43:43-50, 2015.

[11] K C Das, N Akgunes, A S Cevik. On a Graph of Monogenic Semigroups. Journal of Inequalities and Applications, 2013:44, 2013.

[12] N Akgunes, B Cagan. On the Dot Product of Graphs Over Monogenic Semigroups. Applied Mathematics and Computation, 322:1-5, 2018.

[13] H Wiener. Structural determination of paraffin boiling points. J. Amer. Chem. Soc., 69:17-20, 1947.

[14] R C Entringer, D E Jackson, D A Snyder. Distance in Graphs. Czech. Math. J., 26:283-296, 1976.

[15] A A Dobrynin, I Gutman. The Wiener index for trees and graphs of hexagonal systems. Diskretn. Anal. Issled. Oper., 5(2):34-60, 1998.

[16] A A Dobrynin, R Entringer, I Gutman. Wiener index of trees: Theory and applications. Acta Applicandae Mathematicae, 66:211-249, 2011.

[17] A A Dobrynin, I Gutman, S Klavžar, P Ziegert. Wiener index of hexagonal systems. Acta Applicandae Mathematicae, 72:247-294, 2002.

[18] N Akgunes, K C Das, A S Cevik. Some Properties on the Tensor Product of Graphs Obtained by Monogenic Semigroups. Applied Mathematics and Computation, 235:352-357, 2014.

[19] N Akgunes, K C Das, A S Cevik et al. Some properties on the lexicographic product of graphs obtained by monogenic semigroups. J Inequal Appl, 2013:238, 2013.

[20] N Akgunes. Some graph parameters on the strong product of monogenic semigroup graphs. Balıkesir Üniversitesi Fen Bilimleri Enstitüsü Dergisi, 20(1):412-420. 2017.

[21] N Akgunes. Some properties on the disjunctive product over graphs of monogenic semigroups. Advances and Applications in Discrete Mathematics, 19(2):147-159, 2018.

[22] N Akgunes, K C Das, A S Cevik. Topological indices on a graph of monogenic semigroups. Topics in Chemical Graph Theory, 16:3-20, 2014.

[23] N Akgunes, Y Nacaroglu. On The Sigma Index of The Corona Products of Monogenic Semigroup Graphs. Journal of Universal Mathematics, 2(1):68-74, 2019.

[24] N Akgunes. A Further Note on the Graph of Monogenic Semigroups. Konuralp Journal of Mathematics, 6(1):49-53, 2018.

[25] N Akgunes, A S Cevik, I N Cangul. New Indices on Special Graphs. ICRAPAM, 2014 . 